\title{
La sécheresse : conséquences sur le milieu, salubrité, environnement
}

\author{
P. Balland \\ Agence de Bassin Rhône-Méditerranée-Corse, Pierre-Bénite
}

\section{Introduction}

Sous nos latitudes tempérées, la sécheresse est la conséquence de la persistance d'un écart à la "normalité» hydroclimatique, appréciée statistiquement.

Elle n'est pas, chez nous, synonyme d'aridité comme elle l'est dans les pays semi-désertiques ou désertiques, où le concept de normalité hydroclimatique n'a pas réellement de sens.

On en évalue la rareté à travers les notions statistiques de fréquences d'apparition ou durée de retour, et l'intensité par la mesure du déficit hydrique, relativement à la moyenne saisonnière, pour une série de disciplines propres au cycle de l'eau: pluviométrie, hydrométrie, piézométrie...

Cette situation moyenne n'est, bien évidemment, jamais vécue; et l'on relève toujours des variations autour d'elle. Çà n'est que lorsque l'éloignement à cette situation moyenne devient significatif et prolongé qu'il en résulte des conséquences pour les milieux, aux plans quantitatif et qualitatif, et, par suite, sur les usages qu'on peut en faire, quand leur "pouvoir tampon " naturel, c'est-à-dire leur capacité à suppléer, par leurs propres réserves, à un déficit momentané de la ressource, est dépassé.

Un aspect important à considérer du point de vue des effets sur les milieux est la saisonnalité de l'épisode.

\section{Au plan quantitatif}

La sécheresse a bien sûr un impact immédiat, agissant à partir d'un certain seuil, au moment où elle survient, quelle que soit la saison. Elle peut avoir également un impact différé dans le temps, essentiellement s'il s'agit d'une sécheresse froide (se produisant en hiver et/ou au printemps) pour deux raisons principales:

- le déficit du manteau neigeux qui influence le bilan hydrologique global des cours d'eau à régime nival et nivo-fluvial, l'apport de fonte des neiges étant sensiblement réduit

- la recharge insuffisante des aquifères car les pluies ne sont " efficaces " à cet égard que jusqu'à la fin avril ou la mi-mai. Au-delà en effet, le bilan Précipitations - ETP devient négatif, par suite du réveil végétatif et de l'augmentation de l'évaporation due à la remontée de la température de l'air.

La ressource estivale est alors, pour ces deux raisons, globalement déficitaire même si des pluies abondantes se produisent à la fin du printemps.

Cet impact différé est par contre insignifiant pour les sécheresses chaudes, se produisant pendant l'été.

Pour elles toutefois la concomitance avec la canicule aggrave le déficit issu de la seule pénurie des précipitations en raison de l'augmentation du pouvoir évaporant de l'atmosphère (BÉDIOT, 1985).

\section{The drought \\ Consequences on natural surroundings, health and the environment}

A prolonged drought leads to a significant drop in water flow rates in acquatic environments the consequences of which are: an increase in the average concentration levels of pollutants, direct changes in processes such as sedimentation and superficial re-aeration with an impact on acquatic biocoenosis. Linked to possible increases in temperature there may be indirect effects on some chemical processes. Moreover, the reduction in quantities of fresh water can have an effect on some acquatic communities in estuary and lagoon environments and further increase the amount of time the water spends in stagnant environments with possible consequences on the intensity of processes such as eutrophication. Finally, there may be an impact on the quality of underground water following the appearance of conditions that reduce the amount of water exchange with the water nappe. Here we attempt to illustrate these different aspects and examine possible measures to reduce the effects of drought especially as regards pollutant waste. 


\section{Au plan qualitatif}

La vitesse de réchauffement (ou de refroidissement) d'une masse d'eau est, en effet, proportionnelle à la profondeur moyenne. Une réduction du débit d'un cours d'eau consécutive à la sécheresse engendre donc une évolution de la température de l'eau pour deux raisons :

- la diminution de la profondeur moyenne;

- l'augmentation du temps de transit d'une même masse d'eau.

Si l'impact qualitatif est mineur pour les sécheresses froides, il n'en est pas de même pour les sécheresses estivales, le réchauffement de l'eau qui accompagne le déficit de la ressource étant à l'origine d'un cortège d'effets, directs et indirects, sur les processus physicochimiques et biochimiques qui se produisent au sein de la masse d'eau, ainsi, d'ailleurs, que sur les biocénoses aquatiques.

On tente ci-après d'illustrer brièvement certaines des conséquences de la sécheresse vis-à-vis des milieux aquatiques, après avoir rappelé auparavant que l'action de l'homme peut les aggraver ou, au contraire, les réduire.

Elle les aggrave, lorsqu'une politique irréfléchie de déboisement, remembrement mal conduit, pratiques agricoles inadaptées, assèchement de zones humides... est mise en œuvre, qui diminue la capacité de rétention d'eau des sols et, par suite, leur "pouvoir tempon" naturel vis-à-vis d'une anomalie dans le cycle de l'eau. Elle les aggrave aussi lorsqu'une utilisation "vulgaire» de l'eau est faite comme réceptacle et transport de ses déchets.

Elle les réduit au contraire, par une politique d'aménagement des eaux bien conçue permettant de faire face dans une large mesure aux aléas climatiques, consistant à maintenir voire développer cette capacité de rétention des sols ou encore à stocker l'eau en période d'excédent pour la déstocker en période de déficit.

\section{Baisse de la capacité de dilution des milieux}

C'est la conséquence la plus immédiate et " universelle » résultant d'une réduction de la ressource. Elle se manifeste à l'égard des solutés rejetés directement dans les milieux aquatiques. Les substances qui, en un lieu et un temps donnés, "font " la composition chimique d'une eau ont trois origines:

a) géochimique: il n'existe pas dans la nature d'eau chimiquement pure. Toutes les eaux sont "minérales" et naissent d'une roche-mère dont elles s'imprègnent par dissolution et lixiviation. Elles en sont le reflet invariant et vis-à-vis des concentrations des solutés ayant cette origine, la "quantité de ressource" ne joue aucun effet direct.

b) anthropique ponctuelle: les milieux aquatiques reçoivent, véhiculent et transforment les déchets de l'activité humaine. Il y a indépendance entre les flux rejetés et la quantité de ressource réceptrice. Celle-ci exerce vis-à-vis des constituants des rejets un effet de dilution d'autant plus important qu'elle est abondante.
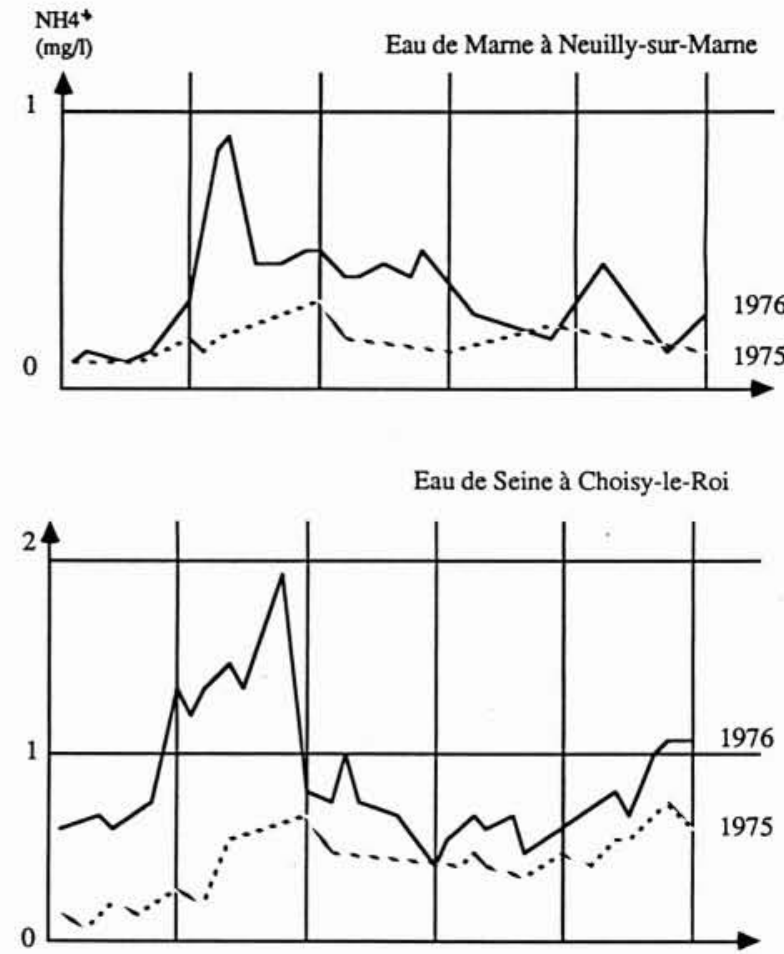

Eau d'Oise à Méry-sur-Oise

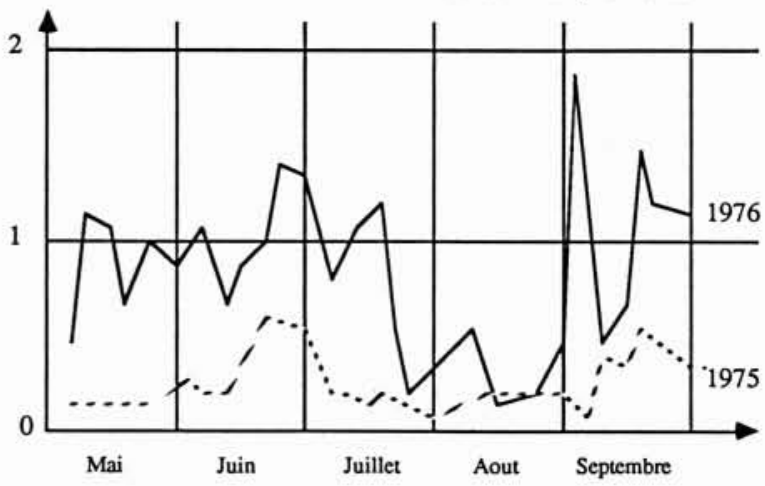

1. Teneur en azote ammoniacal $\left(\mathrm{NH}_{4}^{+}\right)$de l'eau brute de rivière. (Bulletin de liaison $n^{\circ} 33$, déc. 1976, du Comité du bassin Seine-Normandie).

Sa réduction significative et prolongée a pour conséquence une augmentation automatique des concentrations des substances contenues dans les rejets.

Il peut dès lors y avoir dépassement des seuils de tolérance fixés pour un certain nombre d'usages de l'eau : valeurs guides puis valeurs impératives, par exemple, au sens des directives communautaires existantes pour la baignade, la conchyliculture, la vie piscicole, la production d'eau potable, ou objectif de qualité assignés au milieu, sans référence à un usage particulier (cas de certaines substances dangereuses "directivées", ou démarche réglementaire pour l'évaluation de la qualité des eaux...). 
Le dépassement peut être tel que la qualité de l'eau s'en trouve affectée au point d'obérer fortement la pratique de certains usages (interdiction momentanée de la baignade ou de la commercialisation des coquillages, mortalités piscicoles, sur coûts de traitements de potabilisation de l'eau...) ou bien de dégrader de manière intolérable la qualité "patrimoniale» de l'eau et d'en empêcher toute utilisation.

La figure 1 illustre ce phénomène avec l'exemple de l'azote ammoniacal, en 3 points de la Marne, de la Seine et de l'Oise pour la période de mai à septembre des années 1975 et 1976, l'année 1976 ayant été marquée, comme chacun sait, par une très importante sécheresse printanière et estivale.

c) diffuse : origine évoquée ici pour mémoire, dans la mesure où elle est directement dépendante des apports en eau qui "entraînent " dans les milieux récepteurs des substances provenant des terres agricoles et des zones imperméabilisées. Processus à effet de seuil, il ne se manifeste qu'au-delà d'une certaine quantité de ressource.

\section{Conséquences sur les processus physico- chimiques et biochimiques internes aux milieux courants dulçaquicoles}

Elles découlent directement ou indirectement d'une réduction significative et prolongée des paramètres fondamentaux de l'écoulement que sont le débit et la vitesse et, corrélativement, de la "géométrie " de l'écoulement (profondeur, rayon hydraulique, section mouillee) qui est modifiée.

L'ensemble des processus internes aux cours d'eau s'en trouve affecté d'une manière si complexe et intimement liée qu'il n'est pas toujours possible de conclure d'une manière péremptoire sur l'effet de la sécheresse, favorable ou défavorable, vis-à-vis par exemple, du bilan oxygène dissous ou de l'autoépuration. Quatre grands types de phénomènes sont à considérer pour expliciter ceci :

\subsection{Impact direct d'une réduction de la vitesse sur :}

- le transport de la matière organique et les modalités de sa dégradation, dans l'espace et dans le temps. Toutes choses égales, il est simplement possible d'affirmer qu'elle se produit sur un tronçon plus court que dans des conditions "normales » d'écoulement ;

- la sédimentation des fines particules en suspension, favorisée par une réduction de la vitesse, ce qui constitue un terme "puits " dans le bilan global de l'évolution de la manière organique mais altère l'habitat aquatique (cf. infra).Il s'agit au demeurant d'une atténuation toute provisoire d'un des termes du bilan autoépuratoire, car aux premières pluies qui succèdent à un épisode sec prolongé, il se produit une remise en suspension brutale de ces dépôts, auxquels s'ajoutent ceux qui proviennent du lessivage des zones imperméabilisées et des réseaux d'assainissement. Il a bien souvent été démontré que des conséquences dramatiques en résultaient sur la qualité des eaux et des peuplements aquatiques :
- la réaération superficielle

Elle s'exprime comme suit :

$$
\text { Réaération }=K_{2}(C,-C)
$$

$K_{2}$ est le coefficient de réaération superficielle en jour $\left.^{-1}\right), C_{s}$ la concentration à saturation en oxygène dissous à la température de l'eau, et $C$ la concentration observée.

On voit donc que la réaération superficielle est proportionnelle au déficit en oxygène dissous dans l'eau. Ce processus est une bonne illustration ainsi qu'on tente de le démontrer ci-après, de la difficulté de conclure sur l'impact de la sécheresse.

Le coefficient $K_{2}$ s'exprime comme une fonction de la vitesse moyenne $U$ et de la profondeur moyenne $D$ du cours d'eau, de la manière suivante

$$
K_{2}=a U^{h} / D^{\prime}
$$

On voit que, l'effet de la sécheresse agissant dans le même sens sur $U$ et $D$ (toutes deux diminuent), il n'est pas possible de déterminer ses conséquences sur la valeur de $K_{2}$. Celles-ci dépendent de l'évolution respective de ces deux paramètres qui agissent contradictoirement. Les formules les plus couramment utilisées sont les suivantes ( $U$ en $\mathrm{m} / \mathrm{s}, D$ en $\mathrm{m})$ :

\begin{tabular}{ccr}
\hline $\begin{array}{c}U \text { élevée } \\
D \text { élevée }\end{array}$ & Churchill & ${ }^{5,025} \frac{U^{0,969}}{D^{1,673}}$ \\
\hline $\begin{array}{c}U \text { variable } \\
D \text { faible à moyen }\end{array}$ & Owens & $5,34 \frac{U^{0,67}}{D^{1,85}}$ \\
\hline $\begin{array}{c}U \text { variable } \\
D \text { élevée }\end{array}$ & $\begin{array}{c}\text { O'Connor et } \\
\text { Dobbins }\end{array}$ & ${ }^{3,93} \frac{U^{0,5}}{D^{1,5}}$
\end{tabular}

Le diagramme de la figure 2 (page suiv.) fournit la valeur de $K_{2}$ à $20^{\circ} \mathrm{C}$ pour diverses valeurs de $U$ et D.

Remarquant que l'exposant de $D$, la profondeur, est toujours supérieur à celui de $U$, la vitesse, il s'ensuit que :

- si la vitesse baisse moins que proportionnellement par rapport à la profondeur, l'effet sur $K_{2}$ sera à tout coup bénéfique $\left(K_{2}\right.$ augmente) de même que si ces 2 paramètres évoluent à la baisse dans le même rapport ; - l'effet ne sera défavorable que dans le cas où la vitesse baisse plus que proportionnellement par rapport à la profondeur, et seulement jusqu'à une certaine valeur de celle-ci. 


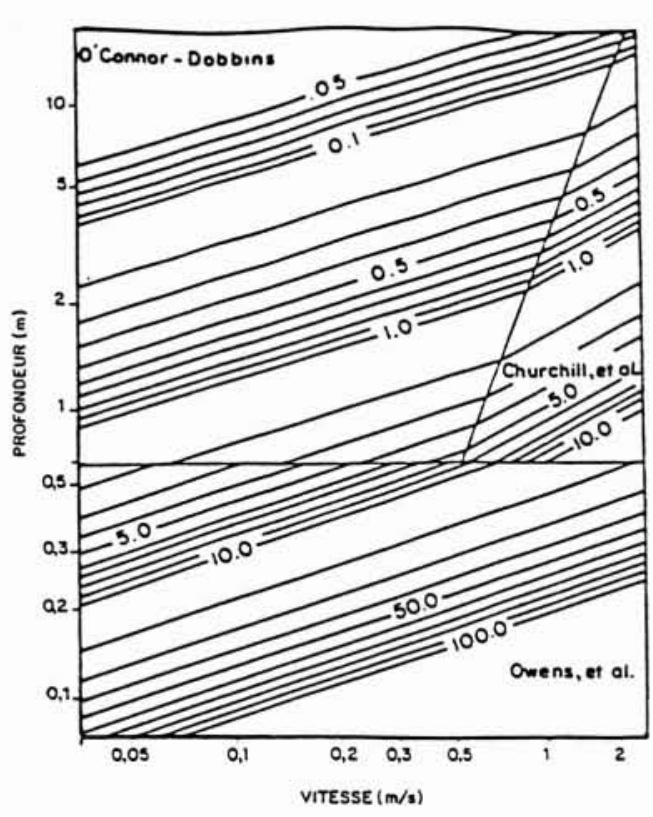

2. Valeurs du coefficient de réaération $K_{2}$ à $20^{\circ} \mathrm{C}$ exprimé en $\left(j^{\prime} \mathrm{r}^{-1}\right)$, en fonction de la vitesse et de la profondeur de l'écoulement en utilisant le découpage de COVAR (1976): trois domaines sont distingués en fonction des conditions expérimentales rencontrées par chaque auteur.

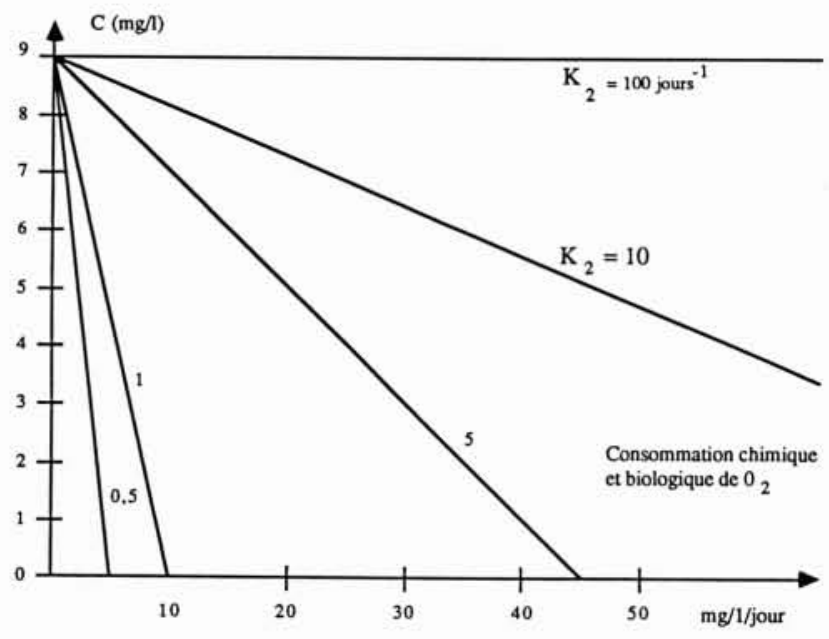

3. Concentration d'oxygène dissous s'établissant dans l'eau ḋ $20{ }^{\circ} \mathrm{C}$ pour différents niveaux de consommation d'oxygène liés à des rejets oxydables et différentes valeurs du coefficient $K_{2}$ d'échange physique d'oxygène à l'interface eau-atmosphère (hypothèse d'absence de production d'oxygène par la végétation).
Exemple: si la vitesse est divisée par 2 par suite de la sécheresse, l'effet sur $K_{2}$ ne sera défavorable que si le coefficient de réduction de la profondeur est au plus égal à

- Churchill : 1,5

- Owens : 1,3

- O'Connor-Dobbins : 1,25 .

On voit l'importance de la géométrie de l'écoulement sur l'appréciation de l'impact sur ce coefficient.

$\mathrm{Au}$ total, cet impact est loin d'être négligeable, compte tenu de l'étendue des valeurs que peut prendre ce paramètre entre 0,1 jour $^{-1}$ (rivière d'environ $5 \mathrm{~m}$ de profondeur à écoulement lent) et 100 jour $^{-1}$ (rivière de profondeur voisine de $0,2 \mathrm{~m}$ à écoulement rapide), ainsi que l'illustre la figure 3 (Gosse et al., 1986).

$\mathrm{Ce}$ coefficient pondère le déficit en oxygène dissous de la rivière, très dépendant de la température, à travers la concentration à saturation $C_{s}$ :

$$
\begin{array}{rll}
\text { à } & 4{ }^{\circ} \mathrm{C} & C_{s}=13 \mathrm{mg} / \mathrm{l} \\
\text { à } 15{ }^{\circ} \mathrm{C} & C_{s}=10 \mathrm{mg} / \mathrm{l} \\
\text { à } 20^{\circ} \mathrm{C} & C_{s}=9 \mathrm{mg} / 1 \\
\text { à } 30^{\circ} \mathrm{C} & C_{s}=7,5 \mathrm{mg} / 1 .
\end{array}
$$

Le réchauffement de l'eau accompagnant une sécheresse estivale influence donc à la baisse (en l'absence de production d'oxygène par la végétation) les stocks d'oxygène dissous présent dans l'eau.

\subsection{Impact indirect d'une réduction de la vitesse et de la profondeur sur le développement végétatif}

La baisse de la vitesse favorise le développement du phytoplancton.

La baisse de la profondeur favorise le développement des végétaux fixés car la lumière peut alors pénétrer jusqu'au substrat. A cet égard, les sécheresses chaudes présentent une nocivité plus marquée que les sécheresses froides.

Le développement explosif de la végétation dans des biefs au cours ralenti est une des principales conséquences d'une sécheresse estivale, ainsi que cela a été démontré à de nombreuses reprises dans le passé.

Ces proliférations induisent des variations nycthémerales extrêmement importantes de la teneur en oxygène dissous et parfois du $\mathrm{pH}$, susceptibles de créer des conditions sublétales voire létales pour de nombreux organismes aquatiques oxyphiles.

On observe notamment :

- une surproduction diurne d'oxygène dissous, accompagnée d'une élévation du $\mathrm{pH}$, due à la photosynthèse. Elle peut atteindre $200 \%$ de saturation;

- à l'inverse une surconsommation nocturne pouvant aller jusqu'au déficit complet, due à la respiration de la matière organique excédentaire et à la décomposition de sa fraction morte. Elle s'accompagne de la baisse du $\mathrm{pH}$. 


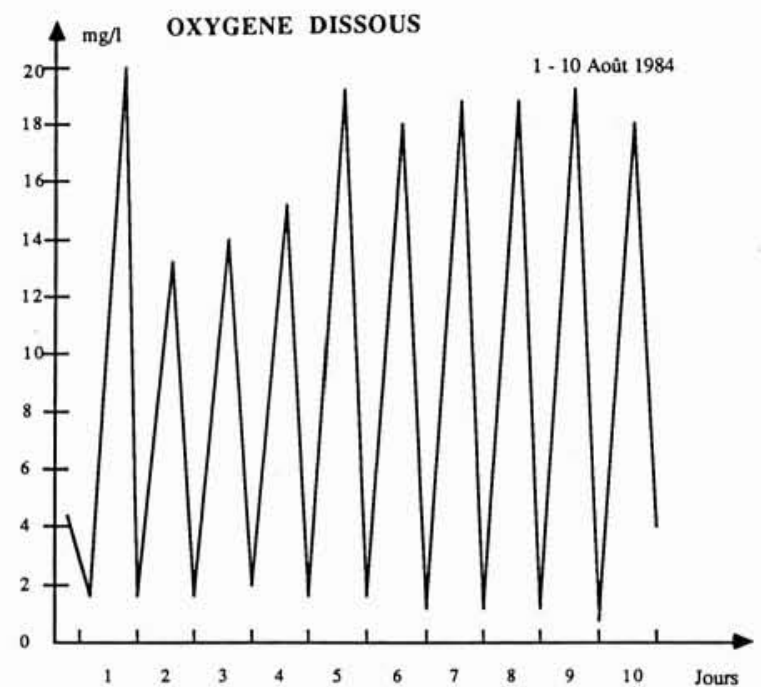

4. Effets de rejets d'éléments minéraux (phosphore, azote), sur la teneur en oxygène dissous du Vair - affluent de la Meuse - en aval de Vittel. Cet apport nutritif permet un développement estival important de végétaux fixés qui expliquent le cycle journalier de l'oxygène dissous du cours d'eau: valeurs élevées de jour, période de production par photosynthèse: valeurs faibles la nuit, période sans production végétale avec mise en évidence de la forte respiration du couvert végétal ; le taux de saturation en oxygène voisin de $0 \%$ au lever du jour peut dépasser $200 \%$ l'après-midi (concentration à saturation voisine de $9 \mathrm{mg} / \mathrm{l}$ ).

Un tel fonctionnement par "à-coup " n'est pas, on l'imagine aisément, compatible avec le maintien d'une faune aquatique de qualité. On a très souvent relevé en période de sécheresse, des mortalités nocturnes de poissons. Par ailleurs, les sursaturations diurnes d'oxygène dissous sont également un facteur de fragilisation des biocènoses pisciaires : l'excès d'oxygène peut en effet brûler le tissu branchial ou provoquer des embolies gazeuses.

La figure 4 illustre cette "cyclicité " particulière de l'oxygène dissous sur le Vair affluent de la Meuse (Gosse et Salleron, 1986).

« Le fait le plus marquant de cette période de sécheresse a été l'aggravation très nette du phénomène d'eutrophisation " (rapport EDF n ${ }^{\circ}$ E31-78 n 19 sur la sécheresse de 1976).

L'altération du cycle de l'oxygène dissous et sa quasiabsence nocturne favorisent les fermentations anaérobies pouvant aller jusqu'à des dégagements gazeux de méthane ou d'hydrogène sulfuré pas franchement sympathiques et responsables d'odeurs nauséabondes.

Par ailleurs, les mauvaises conditions générales du milieu sont propices à la prolifération d'espèces d'algues comme les Cyanophycées et certains champignons Actinomycètes qui s'en satisfont. Ces organismes secrètent des métabolites qui communiquent à l'eau un goût désagréable de moisi (géosmine, isobornéol...). Ceci a été relevé sur de nombreux cours d'eau lors de la sécheresse de 1976 et l'est de manière quasi chronique sur le Doubs (SRAE, 1988), rivière très eutrophe. La relation entre ces développements et la baisse du débit a été établie de façon formelle. Certaines de ces substances peuvent se montrer toxiques vis-à-vis des poissons.

\subsection{Stimulation thermique}

D’une manière générale, en sécheresse estivale, le réchauffement de l'eau ajoute ses effets à ceux d'une baisse des paramètres hydrauliques en stimulant (jusqu'à une certaine limite thermique) l'ensemble des réactions biochimiques qui se produisent dans la masse d'eau. Le métabolisme bactérien est en effet en relation directe avec la température (dans la gamme des valeurs compatibles avec la survie des germes).

Toutes ces réactions font intervenir :

- une cinétique particulière

- un paramètre de pondération.

Si l'on prend l'exemple de la dégradation de la $D B O$, elle s'écrit :

$$
\frac{\mathrm{d}(D B O)}{\mathrm{d} t}=-K_{1}(D B O) .
$$

C'est une cinétique du premier ordre sur laquelle une sécheresse prolongée agit doublement :

- augmentation de la concentration de la $D B O$ par suite de la baisse de la capacité de dilution;

- augmentation de la valeur du coefficient $K_{1}$ de dégradation de la $D B O$, avec la température.

Quelle que soit la réaction biochimique considérée, la relation de son paramètre de pondération avec la température s'écrit

$$
K_{T}=K_{20} \theta^{T-20}
$$

où $K_{T}$ est la valeur du paramètre à la température $T, K_{20}$ sa valeur de référence à $20^{\circ} \mathrm{C}$, et $\theta$ un coefficient supérieur à 1 .

Au total, et toutes choses égales, la dégradation de la $D B O$ par les processus microbiens est accélérée, en condition de sécheresse estivale, par rapport à des conditions normales.

Ceci est vrai pour bien d'autres réactions biochimiques, notamment la nitrification.

En l'absence de facteurs d'inhibition de l'activité microbienne (on verra plus loin qu'il peut s'installer dans le milieu des conditions perturbatrices à cet égard) la sécheresse et le réchauffement stimulent la nitrification. Elle se passe en 2 étapes, de la façon suivante :

$$
\mathrm{NH}_{4}^{+}+\frac{3}{2} \mathrm{O}_{2} \rightarrow \mathrm{NO}_{2}^{-}+\mathrm{H}_{2} \mathrm{O}+2 \mathrm{H}^{+}
$$

germe Nitrosomonas 


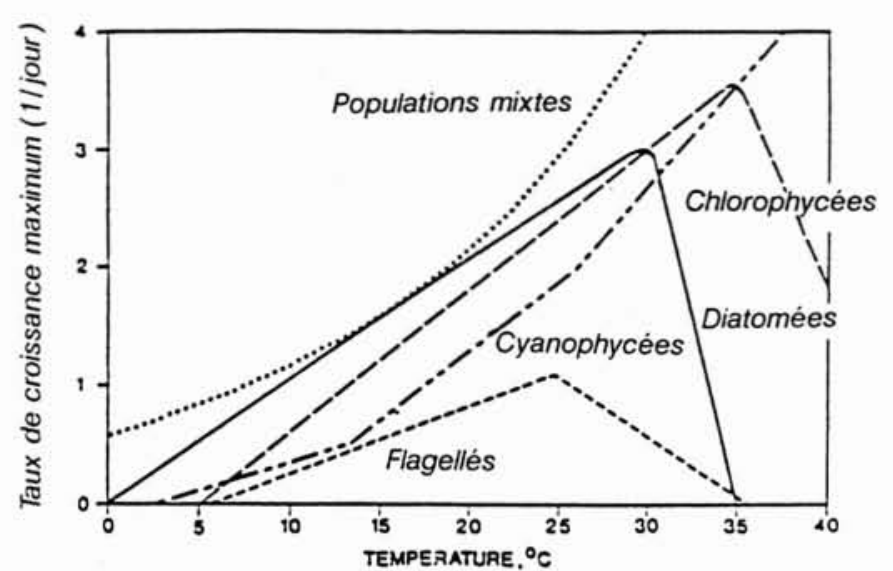

5. Courbe de croissance selon la température pour diverses familles d'algues (Canale, 1974).

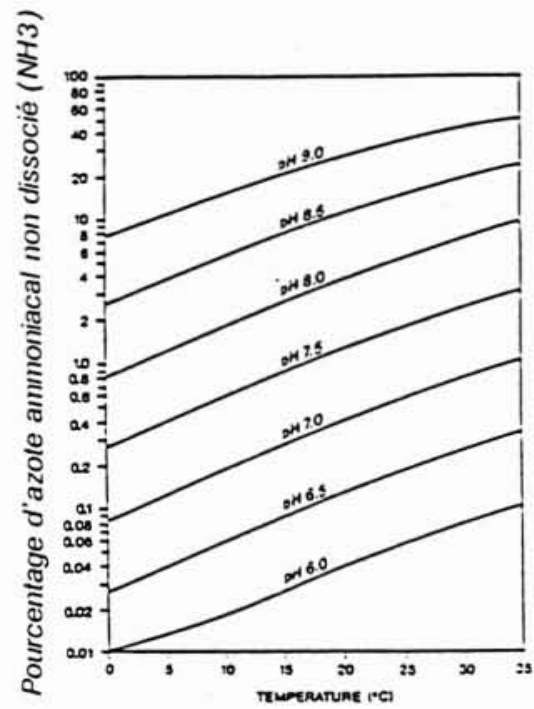

6. Effet du pH et de la température sur l'azote ammoniacal non dissocié.

$$
\frac{\mathrm{NO}_{2}^{-}+\frac{1}{2} \mathrm{O}_{2} \rightarrow \mathrm{NO}_{3}^{-}}{\mathrm{NH}_{4}^{+}+2 \mathrm{O}_{2} \rightarrow \mathrm{NO}_{3}^{-}+\mathrm{H}_{2} \mathrm{O}+2 \mathrm{H}^{+}}
$$

germe Nitrobacter

Du point de vue stœchiométrique, il faut $64 \mathrm{~g}$ d'oxygène pour oxyder en nitrate $14 \mathrm{~g}$ d'azote (ou $4,57 \mathrm{~g}$ $\mathrm{d}^{\prime} \mathrm{O}_{2}$ pour $1 \mathrm{~g}$ de $\mathrm{N}$ ).

La température joue sur ces réactions biochimiques en les stimulant dans la gamme 10 à $30^{\circ} \mathrm{C}$.

Une dernière illustration de la stimulation thermique des processus vivants qui se produisent dans la masse d'eau est son impact sur le taux de croissance de diverses familles d'algues (fig. 5).

Cet effet a une limite supérieure variable selon la famille.

Ceci joue aussi vis-à-vis des organismes pathogènes thermophiles. Les pathologies vis-à-vis des poissons s'en trouvent favorisées.

\subsection{Conséquence des modifications des propriétés de l'eau sur certains processus.}

Les conséquences d'une sécheresse estivale sur les milieux courants peuvent s'enchaîner en cascade. On a vu que la prolifération végétale était favorisée par une sécheresse estivale. Cette prolifération crée de nouvelles conditions de milieu qui, elles-mêmes, engendrent des conséquences sur certains processus : on a à cet égard cité à titre d'exemple, le possible développement de réactions anaérobies au voisinage du fond.

On peut également évoquer à titre d'exemple, l'impact d'une élévation de la température et du $\mathrm{pH}$. sur la dissociation chimique de l'azote ammoniacal. Dans les milieux aquatiques, et selon les valeurs de ces deux paramètres, cet élément peut se présenter sous deux formes :

- la forme dissociée $\mathrm{NH}_{4}^{+}$peu toxique ;

- la forme non dissociée, $\mathrm{NH}_{3}$, extrêmement toxique vis-à-vis de la faune piscicole à des taux aussi bas que $0,005 \mathrm{mg} / \mathrm{l}$.

Le rapport entre ces 2 formes respectives s'écrit :

$$
\frac{\mathrm{NH}_{3}}{\mathrm{NH}_{4}^{+}}=\frac{1}{1+\operatorname{antilog}(10-\mathrm{pH}-0,03 t)} .
$$

Il en ressort que la proportion de $\mathrm{NH}_{3}$ non dissocié toxique est directement fonction du $\mathrm{pH}$ et de la température.

Or la sécheresse estivale favorise le réchauffement de l'eau.

En outre, le développement végétal joue en faveur d'une augmentation du $\mathrm{pH}$, par suite du déplacement qu'il induit de l'équilibre calco-carbonique dans le sens d'une disparition des bicarbonates et du $\mathrm{CO}_{2}$ libre et d'une apparition des carbonates.

Le pourcentage d'azote ammoniacal non dissocié évolue, selon la température et le $\mathrm{pH}$ (fig. 6).

Comparons pour illustrer ceci deux situations, l'une "normale" où le $\mathrm{pH}$ serait de 7 et la température de $18^{\circ} \mathrm{C}$, l'autre, "anormale " où ces mêmes valeurs seraient respectivement 9 et 25 (scénario tout à fait réaliste). Le rapport de non-dissociation $\frac{\mathrm{NH}_{3}}{\mathrm{NH}_{4}^{+}}$évoluerait comme suit :

\begin{tabular}{c|c} 
Situation normale & Situation anormale \\
\hline 0,0035 & 0,35
\end{tabular}


C'est donc par un facteur 100 que ce rapport évolue en faveur de la production de $\mathrm{NH}_{3}$ toxique, avec les conséquences imaginables vis-à-vis de la faune piscicole.

Ce phénomène peut être aggravé par réduction de la nitrification due à des conditions défavorables de milieu créées par la sécheresse $(\mathrm{pH}$ élevés; oxygène dissous déficitaire, présence de composés chimiques inhibiteurs) en dépit de la stimulation de l'activité bactérienne créée par la température, ainsi qu'on l'a évoqué plus haut.

"Les fortes teneurs en ammonium sont liées aux faibles débits et basses oxygénations " (TRAVADE, 1978).

En conclusion de ce bref exposé sur les conséquences possibles d'une sécheresse estivale sur les processus physico-chimiques et biochimiques au sein des milieux courants dulçaquicoles, il apparaît qu'un étiage sévère et prolongé crée des conditions propices à un développement excessif et anarchique de la végétation. Si celui-ci contribue, dans une certaine mesure, à l'autoépuration par la production d'oxygène qui le caractérise, il est à l'origine d'une situation de déséquilibre général de l'écosystème et peut, comme on l'a vu, engendré en cascade une série de nuisances défavorables au maintien d'une vie aquatique de qualité.

Sur le bilan autoépuratoire, différents processus jouent de manière antagoniste ainsi que l'illustre l'équation ci-après d'évolution dans le temps de l'oxygène dissous en une section de cours d'eau.

$$
\frac{\mathrm{d} C}{\mathrm{~d} t}=K_{2}\left(C_{s}-C\right)-K_{1}(D B O)+P-R-\frac{B}{H}
$$

$C=$ concentration en oxygène dissous $(\mathrm{mg} / \mathrm{l})$

$K_{2}\left(C_{s}-C\right)=$ réaération superficielle

$-K_{1}(D B O)=$ dégradation biochimique de la $D B O$

$P$ et $R=$ production et consommation d'oxygène, respectivement, par la végétation $\left(\mathrm{mg} \mathrm{O}_{2} / \mathrm{l} / \mathrm{sec}\right)$

$B=$ demande benthique en $\mathrm{O}_{2}\left(\mathrm{~g} / \mathrm{m}^{2} / \mathrm{s}\right)$

$H=$ profondeur en $\mathrm{m}$.

Le contexte local, on l'a dit, joue un rôle très important sur le bilan global de l'oxygène dissous comme conséquence d'une sécheresse estivale prolongée. Celle$\mathrm{ci}$, au total, joue dans le sens d'une fragilisation et d'un déséquilibre prononcé des milieux courants.

\section{Conséquences de la sécheresse sur l'habitat et la vie piscicole (CEMAGREF, 1989)}

Les modifications de la géométrie de l'écoulement affectent l'habitat aquatique, et la vie piscicole, indépendamment de toute autre considération.

- Le développement de l'herbier aquatique favorise la prolifération d'une faune invertébrée composée d'espèces typiques des milieux benthiques et eutrophes: Hirudinés; larves d'Odonates, Dytiscidés, larves d'Ephémères représentées principalement par des espèces saprobiontes. La réduction de la section mouillée et l'altération de la qualité de l'eau concourent en outre à favoriser les espèces de "poissons blancs" au détriment des salmonidés (truite).

- De plus, les variables physiques de l'écoulement (vitesse, profondeur) ont une importance fondamentale dans la répartition des organismes aquatiques. En effet, la diffusion de l'oxygène dissous, l'évacuation des produits du catabolisme des organismes vivants, le transport des sels nutritifs, la mobilité des organismes, la stabilité du substrat sont sous l'étroite dépendance des vitesses de courant, de la pente de la ligne d'énergie, des hauteurs d'eau, et conditionnent largement le fonctionnement des biocénoses en place.

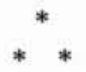

La capacité d'accueil pour les poissons s'en trouve modifiée d'une manière dorénavant quantifiable grâce à différents travaux parmi lesquels:

a) la méthode du Montana : démarche d'expert, dégageant un jugement à partir de l'examen de nombreux cas. A la suite de 17 années d'observations dans les états du Nord de l'Amérique situés entre l'océan Atlantique et les montagnes rocheuses, hydrologues et biologistes ont émis l'hypothèse que pour un débit donné les conditions d'accueil de la faune piscicole étaient caractérisées de façon synthétique par le rapport entre ce débit et le module annuel du cours d'eau, cette dernière valeur étant étroitement liée à la morphologie du cours d'eau.

Le tableau ci-dessous (TENNANT, 1976) résume cette approche.

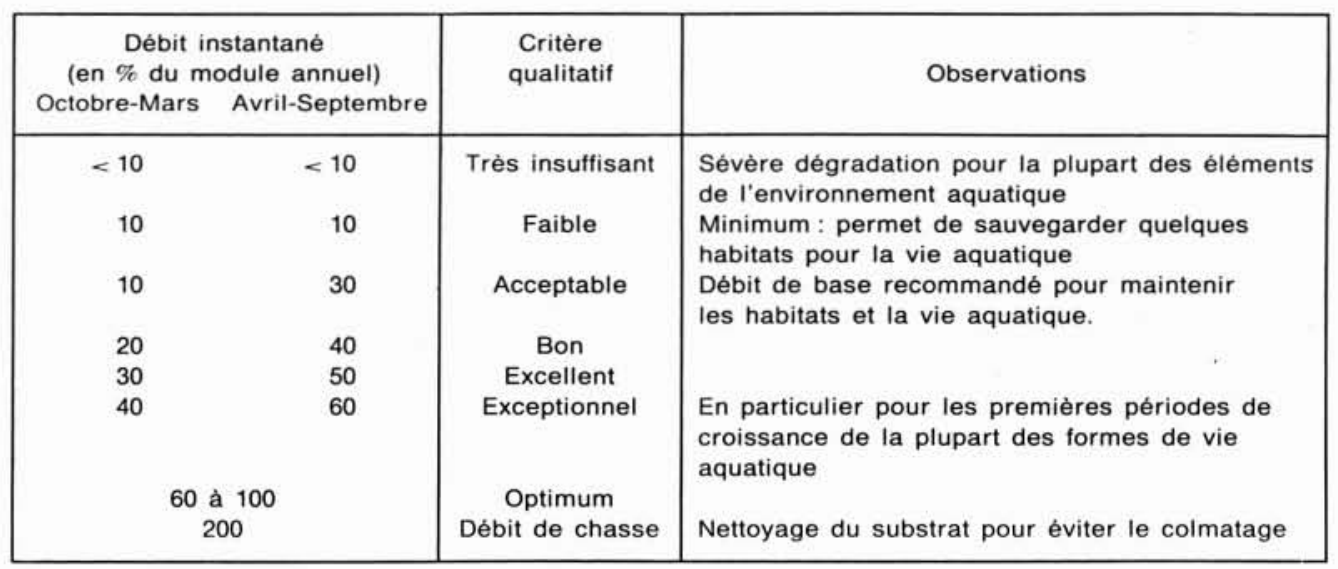




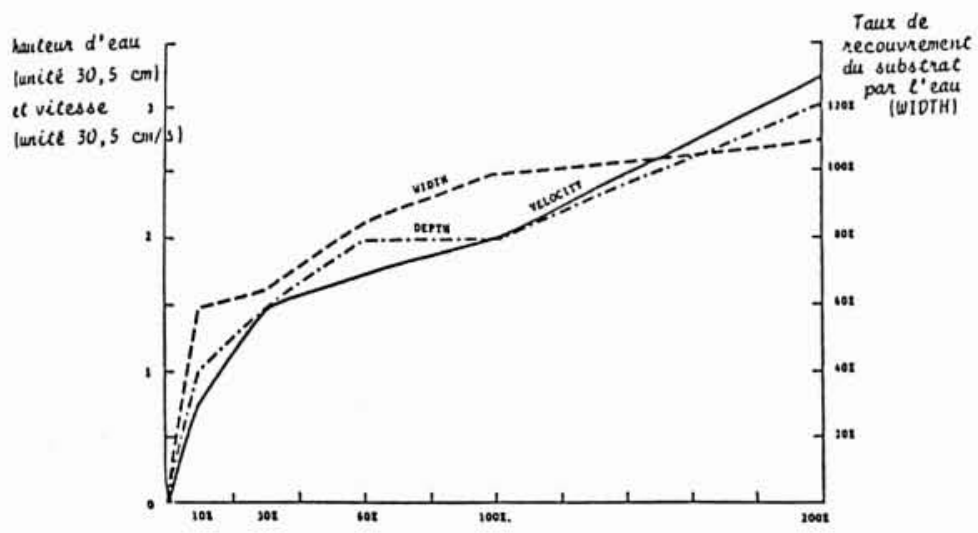

7.

Pourcentage du debit moyen arruel
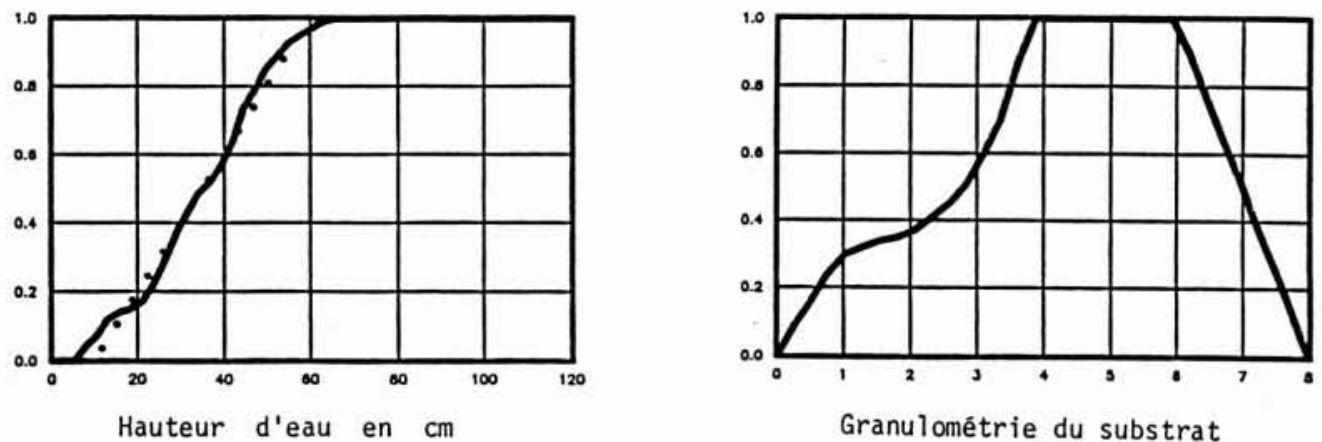

8.

Hauteur d'eau en cm

Granulomētrie du substrat

L'intensité de diverses sécheresses, selon la saison, permet d'en évaluer l'impact sur la vie aquatique.

b) la méthode du "périmètre mouillé» ou de la "conservation des habitats", démarche consistant à cerner l'évolution avec le débit de variables physiques conditionnant le développement des organismes aquatiques. Elle consiste :

- soit à visualiser sur un graphique, à partir de plusieurs observations ou avec l'aide d'un modèle hydraulique, l'évolution de variables globales considérées comme déterminantes pour la capacité d'accueil du cours d'eau vis-à-vis des poissons et à considérer comme valeur de débit critique celle correspondant à une chute drastique de la valeur de ces variables;

- soit à fixer des valeurs limites de vitesses de courant et de hauteurs d'eau dans les seuils et mouilles en deçà (ou au-delà) desquelles certaines fonctions telles que circulation reproduction ou abri des poissons ne peuvent plus être valablement remplies.

Entre 1964 et 1974, le lien a été effectué entre les deux approches: la largeur mouillée, la vitesse moyenne du courant et la hauteur d'eau moyenne ont été mesurées à plusieurs niveaux de débits sur 58 transects situés sur 11 cours d'eau différents des Etats Unis.
L'évolution moyenne de ces paramètres en fonction du pourcentage du module est représentée sur la figure 7 (TENNANT, 1976).

Il s'agit en fait d'une vérification expérimentale de la méthode du Montana. Il apparaît clairement qu'en deçà de $10 \%$ du module, les valeurs des variables mesurées chutent de façon importante, ce qui traduit une modification nette des caractéristiques de l'écoulement et par suite une fragilisation du cours d'eau dont le débit n'est plus en rapport avec la morphologie originelle. Ceci se répercute sur les peuplements piscicoles qui, dans ces conditions, ne trouvent plus les conditions propices à leur maintien.

c) la méthode des microhabitats : démarche explicitant la liaison entre variables physiques et densité d'organismes aquatiques et évaluant la relation entre celle-ci et le débit. Cette liaison quantifiée caractérise la capacité d'accueil du cours d'eau selon le débit. Conçue par un biologiste, Bovée, et un hydraulicien, Milhous, à Fort Collins aux Etats Unis, cette méthode novatrice consiste à coupler :

- un modèle biologique traduisant les relations entre la présence ou la densité relative d'un stade donné d'une 

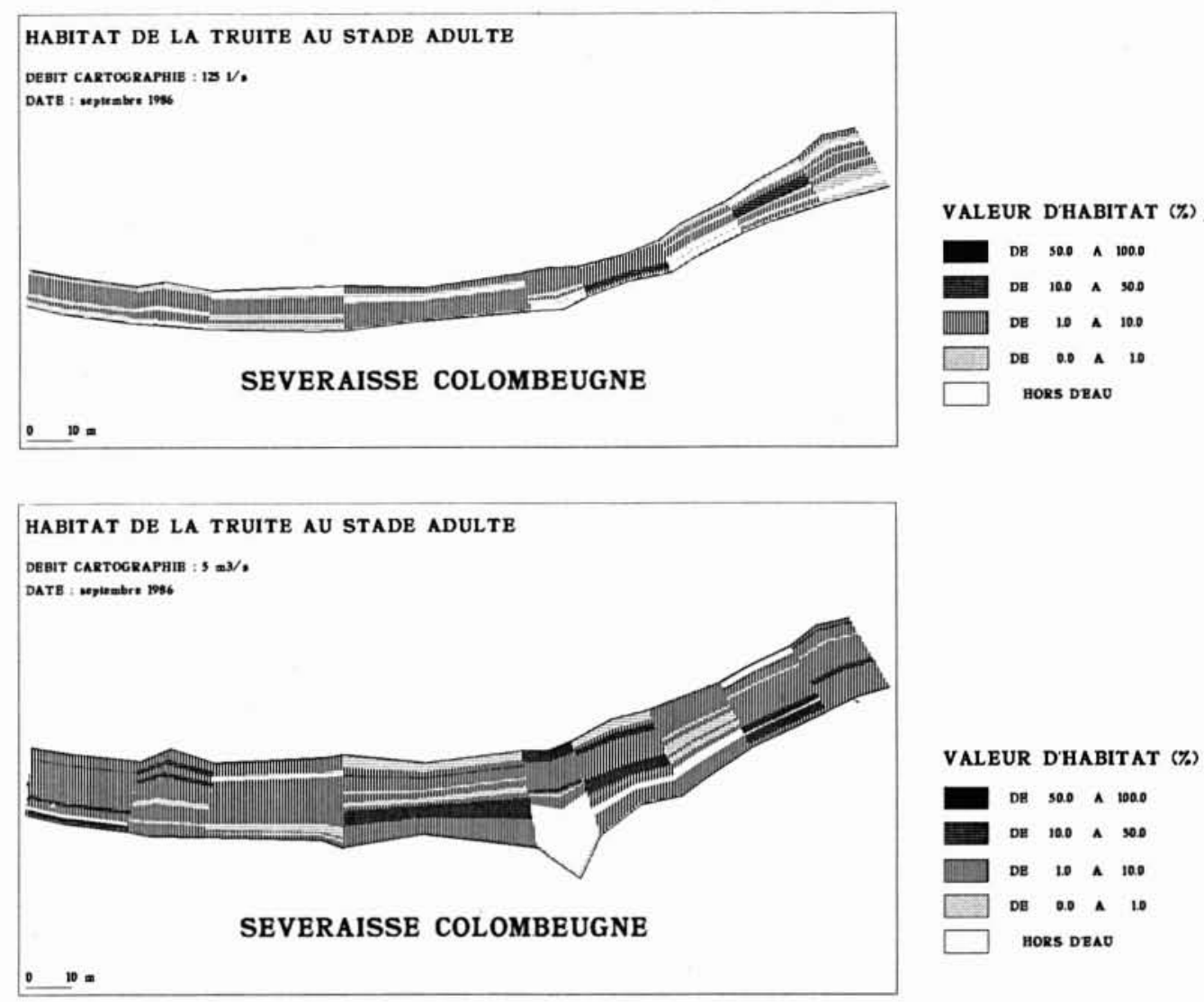

9.

espèce de poisson et les valeurs de plusieurs variables physiques caractéristiques de son habitat ;

- un modèle hydraulique permettant de reconstituer à partir de quelques campagnes d'observations sur un cours d'eau les valeurs des variables physiques retenues, quelque soit le débit transitant dans le secteur étudié.

Chaque observation est considérée comme valable sur l'étendue d'une zone appelée cellule de quelques mètres carrés, située de part et d'autre du transect correspondant. Il est alors possible en utilisant les deux modèles, d'exprimer pour tout débit la capacité d'accueil physique d'une partie de cours d'eau vis-à-vis d'un stade d'une espèce de poisson sous la forme d'une valeur d'habitat au niveau d'une surface pondérée utile qui regroupe plusieurs cellules homologues. Les variables prises en compte pour l'évaluation de la valeur d'habitat sont la hauteur d'eau, la vitesse du courant et la granulométrie du substrat.

Chacune d'elle s'exprime au travers d'une fonction de préférence, courbes dont la valeur s'étage ente 0 et 1 , la valeur maximale étant obtenue pour la valeur de la variable physique correspondant à l'optimum du " confort" du poisson.
L'adéquation des conditions du milieu par rapport aux exigences de l'espèce considérée, c'est-à-dire la valeur d'habitat pour celle-ci, s'exprime par le produit des trois fonctions de préférence. Ainsi si une seule de celle-ci est nulle, la capacité d'accueil ou valeur d'habitat l'est aussi. A l'inverse, si ces trois fonctions sont maximales (égales chacune à 1), la valeur d'habitat est aussi maximale (et égale à 1 ).

On verra sur la figure 8 la représentation graphique de deux de ces fonctions de préférence, relatives à la hauteur d'eau et à la granulométrie du substrat pour la truite fario adulte (courbes Bovée, revues CEMAGREF).

Le modèle hydraulique est un modèle classique unidimensionnel de calcul de ligne d'eau.

Le couplage des deux modèles permet de cerner l'évolution de la surface pondérée utile d'une station représentative d'un secteur de cours d'eau en fonction du débit et pour différents stades de vie d'une espèce de poisson.

L'application à un tronçon de la rivière Severaisse, dans le département des Hautes Alpes pour deux valeurs extrêmes de débit, respectivement $125 \mathrm{l} / \mathrm{s}$ et $5 \mathrm{~m}^{3} / \mathrm{s}$ est donnée dans la figure 9. 
L'approche a permis de montrer une bonne concordance entre biomasse de truites fario adultes et surfaces pondérées utiles critiques, ce qui permet d'envisager l'utilisation de la méthode des microhabitats pour prévoir l'évolution d'un stock de truites en fonction du débit.

On voit tout l'intérêt de cette méthode pour prévoir l'impact d'une sécheresse prolongée sur les peuplements, d'un point de vue purement physique. Bien entendu, cet impact ajoute ses effets à ceux d'une dégradation qualitative du milieu.

\section{Conséquences de la sécheresse sur les écosystèmes lagunaires du littoral méditerranéen (FRISONI, 1989)}

Les lagunes du littoral méditerranéen occupent une position intermédiaire entre la mer avec laquelle elles communiquent de façon plus ou moins permanente et les bassins versants côtiers dont elles constituent fréquemment l'exutoire des eaux superficielles et mêmes souterraines.

Cette position particulière participe de manière prépondérante au fonctionnement et à l'organisation de ces écosystèmes spécifiques. Il est alors intéressant de s'interroger sur le devenir de tels milieux dans le cas d'une réduction des apports liés à l'installation provisoire ou durable de conditions climatiques caractérisées par de faibles précipitations - période de sécheresse.

\subsection{Le fonctionnement général et l'organisation biologique des lagunes littorales}

Le fonctionnement hydraulique et hydrologique des lagunes littorales est soumis à la double influence des eaux douces issues du bassin et des eaux marines pénétrant par les graus. La salinité particulièrement fluctuante des eaux lagunaires traduit cette double influence. La circulation des eaux (hydrodynamisme) au sein des plans d'eau est pour sa part tributaire des apports périphériques, des marées (généralement faibles en région méditerranéenne) mais surtout des vents. Le régime thermique des eaux est pour sa part sous l'étroite dépendance des variations saisonnières de la température et de l'ensoleillement. Ainsi le climat joue un rôle majeur dans l'évolution des caractéristiques physiques et climatiques du domaine lagunaire. Cette évolution est marquée par une très forte variabilité :

- les apports en provenance du bassin versant dépendent directement des précipitations, celles-ci sont particulièrement fluctuantes d'une année sur l'autre (200 à $1000 \mathrm{~mm} / \mathrm{an})$. Elles surviennent généralement entre l'automne et le printemps au cours de brefs épisodes, pouvant dépasser en 24 heures le dixième de leur valeur annuelle. Les apports sont ainsi irréguliers, souvent brutaux, et parfois absents pendant de longues périodes de sécheresse s'étalant sur plusieurs mois. Le débit du Réart, affluent de l'étang de Canet dans les Pyrénées Orientales, varie entre 0 et $350 \mathrm{~m}^{3} / \mathrm{s}$;
- les échanges avec la mer dépendent de la nature des communications (graus) souvent obstruées par les sédiments côtiers, du régime des vents et de celui des marées. Les vents violents (mistral, tramontane, marin...) provoquent une bascule des plans d'eau et engendre au travers des graus un courant dont le sens dépend du dénivelé entre mer et étang. Les marées semidiurnes (ou lunaires) régulières, mais de faibles amplitudes $(15 \mathrm{a} 30 \mathrm{~cm})$ ont un effet limité sur le mouvement des masses d'eau. Les marées barométriques, pour leur part, sont plus importantes (40 à $60 \mathrm{~cm}$ ) elles interviennent lors de l'installation de conditions météorologiques (dépression, anticyclone) qui favorise l'existence d'une différence importante de niveau entre mer et étang. Souvent associées à des vents violents elles sont à l'origine d'échanges hydrauliques plus importants entre mer et étang. Entre l'étang de Thau (Hérault) et la mer, la marée semi-diurne est à l'origine d'échanges compris entre 0,7 et 3,7 millions de $\mathrm{m}^{3}$ alors que ceux-ci peuvent atteindre 27 millions de $\mathrm{m}^{3}$ sous l'effet d'une forte marée barométrique.

Ainsi le régime hydrologique et hydrodynamique d'un étang littoral méditerranéen est soumis à des logiques ayant en commun un caractère fugace, aléatoire, exceptionnel voire catastrophique : crues sur le bassin versant, vents violents, fortes marées barométriques.

L'organisation biologique au sein des lagunes dépend de nombreux facteurs édaphiques parmi lesquels interviennent en particulier la salinité des eaux et le degré d'échanges avec la mer.

La salinité des eaux résulte du bilan hydrique général (apports du bassin versant-échanges avec la mer-évaporation). Compte tenu des caractéristiques du régime hydrologique précédemment décrit, la salinité des eaux est particulièrement variable tant en ce qui concerne sa répartition spatiale au sein de l'étang qu'en ce qui concerne son évolution saisonnière et même annuelle. En conséquence, les espèces qui colonisent ces milieux sont en général marquées par une forte euryhalinité (aptitude à supporter les variations de salinité). Ainsi les conditions changeantes de salinité (voir d'autres paramètres comme la température ou l'oxygénation des eaux) peuvent ponctuellement affecter le développement de tel ou tel organisme, mais remettent rarement en cause leur présence à long terme dans la lagune. Les phénomènes de recolonisation du milieu sont en général très rapides dès que l'épisode responsable de la disparition d'organismes vivants a cessé.

La répartition des espèces et des peuplements biologiques dans les lagunes est sous la dépendance du degré de relation avec la mer. A proximité des communications entre mer et étang se rencontrent une faune et une flore riche et diversifiée à forte affinité marine. Au sein des lagunes, au fur et à mesure de l'éloignement de la mer (que les auteurs cités appellent "le confinement»), se développent des peuplements moins riches, mais souvent plus productifs, composés d'espèces ou de variétés très euryhalines que l'on ne rencontre qu'en lagune.

Généralement, les peuplements rencontrés en milieux dessalés et en milieux salés, présentent de ce fait de fortes similitudes. 
Il faut atteindre les confins les plus éloignés de l'influence marine pour distinguer selon les caractéristiques hydroclimatiques du milieu soit des faunes à forte affinité dulçaquicole, dans les zones - ou en période d'apport prépondérant du bassin versant, soit des faunes caractéristiques des milieux aquatiques temporaires et sursalés, dans les zones évaporitiques.

Cette zonation biologique est donc considérée comme dépendante directement du degré de relation avec la mer. Les apports d'eau douce, plus que la salinité ellemême, agissent comme une "entrave " à la pénétration des courants marins qui fournissent aux peuplements lagunaires les éléments vitaux nécessaires à leur développement.

A la périphérie des plans d'eau littoraux se développent des associations végétales (roselières, sansouires, enganes, prés salés...) dont la présence et la répartition dépendent de la salure des sols et des eaux d'une part, de la fréquence et du niveau de submersion d'autre part.

\subsection{Analyse théorique et illustration de l'impact de la sécheresse sur le fonctionnement et l'organisation biologique des écosystèmes lagunaires}

L'absence durable de précipitations sur le bassin versant aura pour effet direct de diminuer les apports d'eau douce transitant habituellement vers l'étang.

Sur un plan d'eau en communication permanente avec la mer, cette diminution de l'influence des eaux douces peut entraîner une "marinisation" du milieu se traduisant par une extension des zones colonisées par les peuplements à affinité marine ou lagunaire.

C'est ainsi que les herbiers à Potamogeton et Characées des lagunes littorales sont susceptibles de véritables régressions comme cela a été observé sur les étangs de l'Or (Hérault), Bages-Sigean (Aude) ouBiguglia (Corse). Dans le même temps, ces milieux connaissent une extension des zones colonisées par des Phanérogames à plus forte affinité marine tels les Ruppia ou les Zostères. Cette évolution est généralement réversible en cas de rétablissement des apports d'eau douce. De telles successions ont déjà été observées à Bages-Sigean ou à Biguglia. Le remplacement d'un type d'herbier par un autre n'est cependant pas immédiat et l'on observe souvent une période transitoire au cours de laquelle les fonds restent dénudés modifiant totalement les conditions d'accueil des biocénoses animales. Un impact socio-économique peut être enregistré lorsqu'une partie du peuplement animal concerné par la modification du biotope, fait l'objet d'une exploitation par l'homme. Ce peut être le cas des anguilles dont la pêche est surtout développée dans les parties les moins salées des milieux lagunaires. Dans le cas de la conchyliculture, l'absence d'apport nutritif véhiculé par les eaux douces peut limiter les floraisons phytoplanctoniques, base de l'alimentation des mollusques filtreurs mis en élevage.

Cette période transitoire de déséquilibre peut voir le développement temporaire d'autres végétaux — algues macrophytes (ulvacée par exemple) ou microphytes (cyanophycées) - lorsque les conditions nutritives du milieu leur sont favorables. Lorsque ces conditions se maintiennent (eutrophisation liée à l'activité humaine périphérique par exemple) ces algues, en particulier les macrophytes, peuvent définitivement occuper le milieu empêchant ainsi le retour des peuplements végétaux originels.

Dans les milieux en communication temporaire (ou limitée) avec la mer, la diminution des apports en provenance du bassin versant entraîne un réel déficit hydrique aboutissant généralement à la sursalure des eaux. Les biocénoses en place peuvent alors évoluer radicalement: disparition des peuplements aquatiques dulçaquicoles, apparition de peuplements caractéristiques des milieux évaporitiques.

A la périphérie des étangs, les périodes de sécheresse favorisent l'apparition de changements importants dans la végétation. L'absence de submersion par l'eau douce permet une remontée des nappes salées d'origine lagunaire ou marine favorisant la salure des sols et le développement de végétaux halophyles (salicornes par exemple). L'absence de submersion de quelques origine qu'elle soit (eau douce, eau saumâtre), empêche le développement de certains végétaux. On observe ainsi fréquemment en période de sécheresse la régression des roselières périphériques des étangs littoraux et leur remplacement par des sansouires.

Ce phénomène est accentué par l'activité agricole des plaines littorales qui nécessite en période de sécheresse, le prélèvement par pompage des eaux douces véhiculées par les réseaux de drainage. Lorsque ces phénomènes entraînent une remontée de la nappe salée, le retour aux conditions originelles ne sera effectif qu'après de longues périodes pluvieuses nécessaires au lessivage des sols salés. C'est ainsi que la succession d'années sèches et/ou la modification de la gestion hydraulique des réseaux de drainage à des fins agricoles peuvent être mises en cause pour tenter d'expliquer les régressions de roselières observées sur l'étang de l'Or (Hérault) ou l'étang de Campignol (Aude).

Compte tenu de la fonction d'habitat jouée par le couvert végétal vis-à-vis de l'avifaune, la sécheresse a des répercussions sensibles en ce qui concerne l'accueil, le nourrissage ou la reproduction des oiseaux fréquentant le milieu lagunaire.

La diminution des apports en provenance du bassin versant peut entraîner une diminution du taux de renouvellement des étangs littoraux, y favorisant ainsi la stagnation des masses d'eau et les phénomènes d'accumulation (matière organique par exemple). Ainsi l'apparition de crises dystrophiques (malaigues) au cours de l'été dans les étangs littoraux peut être partiellement attribuée à la faiblesse des pluies printanières. En Languedoc-Roussillon, les crises survenues au cours des étés entre 1975 et 1987 étaient souvent consécutives à des printemps particulièrement secs (précipitation inférieure à $160 \mathrm{~mm}$ ) par rapport à une moyenne établie sur 43 ans (202 mm). Au cours de la période 1945-1987, la sécheresse relative de l'épisode postérieur à 1975 (moyenne $=709 \mathrm{~mm} /$ an avant $1975,660 \mathrm{~mm} /$ an après 1975) pourrait être mise en cause pour expliquer le caractère particulièrement étendu des crises dystrophiques survenues depuis 1975.

Cette hypothèse soulève cependant un paradoxe dans la mesure où l'eutrophisation, cause présumée des crises 


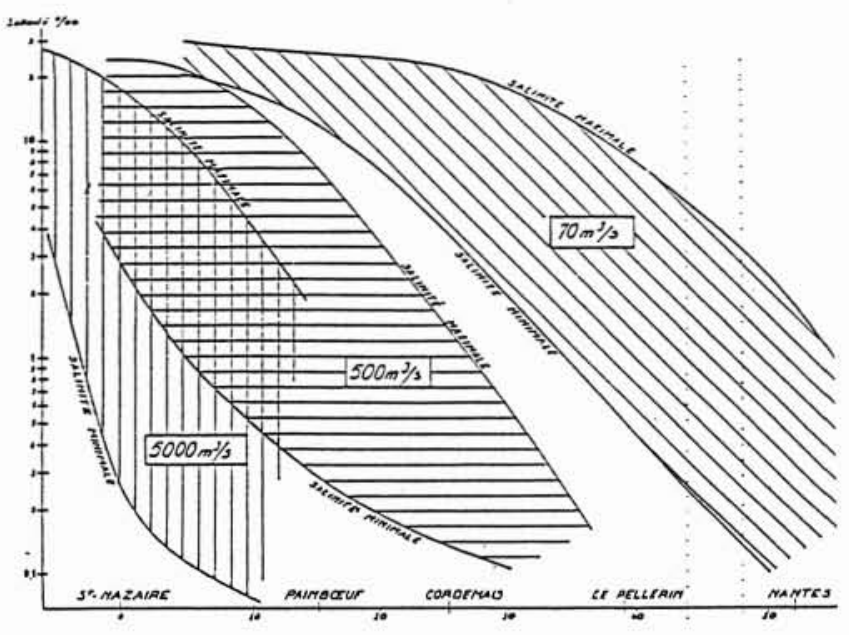

10. Evolution des salinités maximales et minimales en fonction du débit de la Loire.

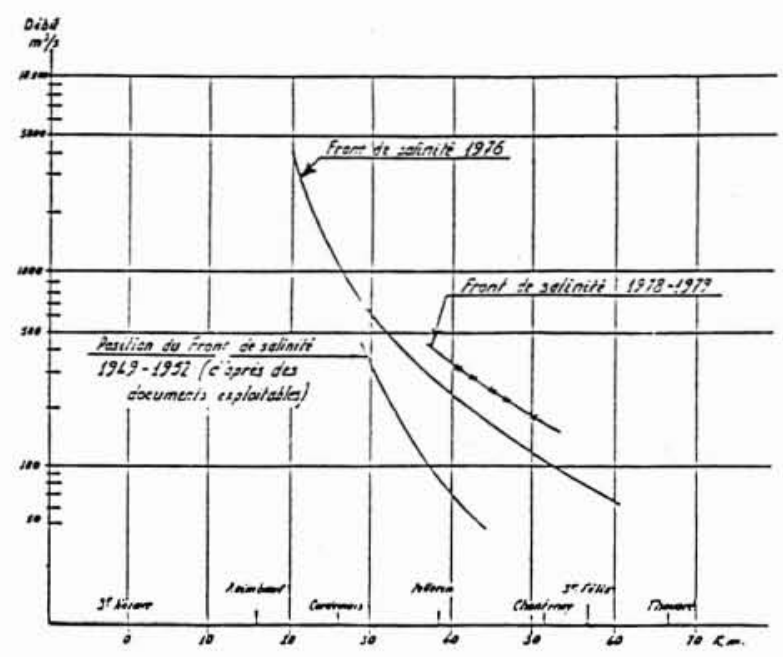

11. Limites du front de salinité $(0.5 \%)$ en fonction du débit en vive-eau. dystrophiques, est fortement liée à l'enrichissement en sels minéraux et en matière organique dû aux apports du bassin versant. Le développement de l'urbanisation à la périphérie des étangs depuis les années 60 intervient donc probablement dans l'apparition de ces crises estivales.

\subsection{Conclusion}

L'évolution, le fonctionnement des lagunes littorales sont régis par des facteurs (climatiques, hydrologiques) particulièrement fluctuants d'une année (ou d'une saison) à l'autre.

La faune et la flore qui peuplent ces écosystèmes sont composées d'espèces généralement eurybiontes adaptées aux variations de salinité des eaux ou au caractère temporaire de certains plans d'eau.

Les périodes de sécheresse qui affectent l'environnement lagunaire font aussi partie du cortège d'influences diverses (crues, vents violents, situations dépressionnaires) aléatoires, à caractères " catastrophiques " qui agissent sur les écosystèmes sans remettre en cause leur pérennité.

A ce titre, l'impact d'une période de sécheresse se traduit par des successions de biocénoses dont le caractère est rarement irréversible.

Ces phénomènes provoquent des modifications fugaces mais dont les répercussions socio-économiques sont cependant importantes (diminution des ressources halieutiques, aquacoles ou cynégétiques). Par ailleurs, les périodes de déséquilibre qui s'instaurent au cours des évolutions observées peuvent créer les conditions favorables à une véritable dégradation du milieu dans le cas où d'autres phénomènes plus durables leur sont associés (eutrophisation liée aux rejets urbains, intensitifcation des prélèvements d'eau pour l'agriculture...).

\section{Conséquences de la sécheresse sur les milieux estuariens}

La réduction des apports d'eau douce en liaison avec la sécheresse influence, avec d'autres facteurs, comme l'amplitude de la marée et la géométrie, la pénétration de l'eau salée dans un estuaire (BRosSARD et GALLENNE, 1982).

Dans le cas de l'estuaire de la Loire, il est possible de représenter, selon le débit, l'intrusion saline (voir fig. 10).

Le front de salinité, caractérisé par une teneur de $0,5 \%$ progresse ainsi vers l'amont en vive eau d'environ $20 \mathrm{~km}$ pour un débit de la Loire de $70 \mathrm{~m}^{3} / \mathrm{s}, 10 \mathrm{~km}$ pour $200 \mathrm{~m}^{3} / \mathrm{s}, \ldots$ (fig. 11).

Il en résulte en période d'étiage, des teneurs élevées en chlorures au niveau des prises d'eau potable; durant l'étiage de 1976, avec un débit exceptionnel de $70 \mathrm{~m}^{3} / \mathrm{s}$, on a relevé au niveau de la prise d'eau de la ville de Nantes, des teneurs voisines de $500 \mathrm{mg} / \mathrm{l}$ de $\mathrm{NaCl}$ au moment de la pleine mer pendant quelques minutes.

Les variations de salinité dans l'estuaire s'accompagnent également de variations parallèles des principaux constituants de l'eau de mer ( $\mathrm{Ca}, \mathrm{Mg}, \mathrm{K} . .$.$) .$

Les fluctuations de salinité dans le temps et l'espace contribuent en outre à l'établissement de processus complexes de dilution, diffusion, dispersion des éléments chimiques, qui eux-mêmes influencent les réactions biochimiques et la nature des peuplements planctoniques 
et microbiologiques, spécifiques de ces milieux particuliers.

Le bouchon vaseux, dont l'existence est permanente, représente la zone où la concentration de matières en suspension est plus élevée (de $100 \mathrm{mg} / \mathrm{l}$ à quelques grammes/l) que celle des eaux fluviales ou marines (inférieur à $50 \mathrm{mg} / \mathrm{l}$ ). A chaque marée il oscille dans l'estuaire. Il subit en outre des oscillations saisonnières importantes, liées aux apports d'eau douce.

Pendant l'étiage exceptionnel de $1976\left(70 \mathrm{~m}^{3} / \mathrm{s}\right)$, sa partie amont a atteint Thouaré, c'est-à-dire à près de $70 \mathrm{~km}$ de l'embouchure. En étiage "normal " (100 à $200 \mathrm{~m}^{3} / \mathrm{s}$ ), il s'étale entre Paimbœuf et Nantes. En période de crue, il est "expulsé " en baie de Bourgneuf. Il influence directement, par sa teneur élevée en matière organique, l'évolution de l'oxygène dissous.

La remontée de l'intrusion saline au cours des dernières années dans l'estuaire de la Loire a entraîné certaines difficultés pour l'approvisionnement en eau douce nécessitant de prévoir une gestion des installations de prise en fonction de la fluctuation de la salinité en tenant compte du débit du fleuve, de la marée et de la profondeur de prélèvement.

Les usages agricoles (prés salés de Loire) et industriels s'en sont également trouvés affectés.

De même l'extension du bouchon vaseux et ses oscillations s'accompagne d'un risque d'envasement des installations de prise d'eau. Dans le cas de l'alimentation en eau de la ville de Nantes, ces différents aléas dus pour partie à la sécheresse ont conduit à reporter en amont la prise d'eau. Dans un autre contexte, celui des rivières de Charente et de Seudre et du Bassin de Marennes Oléron intensivement utilisé par l'ostréiculture, la sécheresse de 1976 s'est traduite par une très forte dégradation de la qualité du milieu et un développement de l'eutrophisation (HÉrAl et al., 1978). Le développement du dinoflagellé Prorocentrum micans en particulier a été spectaculaire. En conditions " normales ", il est quasiment absent de ce milieu. Il libère à sa mort une toxine pouvant être à l'origine de mortalités des bivalves. La réduction des apports d'eau douce, accompagnée d'une dégradation de la qualité, a èté à l'origine, en Seudre, d'importantes mortalités de l'huître Crassostrea gigas et de poissons. Les émissions de naissain ont en outre été fortement affectées par les mauvaises conditions ambiantes.

\section{Conséquences de la sécheresse sur les eaux souterraines}

L'alimentation des nappes d'eau souterraine dépend, comme celle des rivières, de l'efficacité des pluies, c'està-dire de leur fraction non évaporée qui atteint la surface du sol. (CAStany et Margat, 1976). Mais la pluie efficace pour l'infiltration n'est pas proportionnelle à la pluie tombée. Elle varie beaucoup selon les époques de l'année. Ainsi, il est constaté que la recharge des aquifères, très sensible à la distribution saisonnière des pluies, se produit pour l'essentiel entre octobre et mars. Il en résulte que la sécheresse, définie comme un déficit des précipitations par rapport à la normale, a sur le régime des nappes d'eau souterraines deux effets bien distincts selon la saison durant laquelle elle sévit:
- la sécheresse d'hiver se traduit par un défaut d'alimentation naturelle des nappes par l'infiltration des eaux de pluie ;

- la sécheresse d'été, si elle a des conséquences très préjudiciables pour l'agriculture par le dessèchement des sols, n'a par contre aucun effet direct sur les eaux souterraines puisque celles-ci même en année normale ne sont pas (ou très faiblement) alimentées par l'infiltration des pluies estivales.

En revanche surtout si la sécheresse se prolonge en début d'automne, le dessèchement des sols, plus accentué, peut retarder et restreindre les apports par infiltration au cours de la saison pluvieuse suivante. En outre, le défaut d'alimentation des rivières par tarissement du ruissellement, aggravant la faiblesse des débits d'étiage d'été, accentue la baisse de la cote de l'eau dans leur lit. Il en résulte une vidange, accrue dans le temps, des réserves d'eau souterraine des nappes alluviales liées aux cours d'eau, donc une baisse de leur niveau, plus ample et plus précoce qu'en année normale. Autrement dit, la sécheresse diminue les recettes et accroît les dépenses en eau des réservoirs aquifères. Ces deux actions, plus ou moins synchrones, concourent à l'abaissement des niveaux des nappes. Toutefois, les aquifères, à la différence des cours d'eau, sont non seulement des systèmes conducteurs, mais aussi des réservoirs dotés d'une capacité plus ou moins grande, mais très supérieure généralement aux possibilités de stockage des réservoirs de surfaces naturels (lacs) ou artificiels (retenues de barrages). Les volumes d'eau emmagasinés en moyenne dans beaucoup de grandes aquifères régionaux sont souvent supérieurs à cinq ou dix fois ceux reçus ou débités annuellement en moyenne par ces réservoirs. Cette durée de renouvellement, c'est-à-dire le temps qui serait nécessaire pour renouveler totalement la réserve si elle était épuisée, leur confère une faible sensibilité aux effets d'une année sèche isolée.

Une série d'années sèches consécutives ( 3 ou 4 ) est en général nécessaire pour provoquer des baisses notables des niveaux des nappes et par conséquent des augmentations de la profondeur de l'eau, voire le dénoyage des puits et des décroissances exceptionnelles des débits des sources. Il n'y a donc pas de relation directe, à court terme, entre les niveaux d'étiage des nappes et les précipitations de l'année considérée.

Cette sensibilité, ou vulnérabilité, des nappes d'eau souterraines à la sécheresse est toutefois variable selon les caractéristiques des réservoirs aquifères: extension, épaisseur, emmagasinement, perméabilité, degré de liaison avec les cours d'eau, profondeur de la surface libre des nappes, etc... Localement il peut exister des situations très vite critiques sur de petits aquifères alluviaux ou de socle. Ainsi, la sécheresse très accentuée de 1976 , mais isolée, non consécutive à des années sèches antérieures, n'a pas provoqué de conséquences notables sur les grandes nappes. En revanche, elle a affecté des aquifères locaux de faible capacité. Tel a été le cas des régions de l'ouest à nappes nombreuses mais de faible extension et de capacité limitée. Ainsi dans le Massif Armoricain, de nombreuses petites sources habituellement pérennes ont tari.

Elle a un effet secondaire mais plus immédiat, sur les nappes alluviales très liées à des rivières. En effet, la 
faible hauteur d'eau dans les cours d'eau a provoqué un abaissement des niveaux de ces aquifères de faible capacité en général et d'extension latérale limitée. Celuici a entraîné la décroissance des productivités des nombreux captages exploitant ces aquifères. Cette baisse s'est traduite soit par une diminution des quantités d'eau fournies, soit par une augmentation du coût de leur production, à débit égal, du fait de la nécessité de pomper à des profondeurs plus grandes. Il a été parfois nécessaire de forer des puits supplémentaires pour prélever le même débit total dans les champs de captage. Les besoins accrus de l'agriculture ont en outre parfois, amplifiés les prélèvements. Localement, la baisse saisonnière des niveaux des nappes sollicitées s'en est trouvée ainsi augmentée.

La surexploitation, en période de sécheresse, de certains aquifères alluviaux peut inverser le sens naturel de l'écoulement de l'eau souterraine normalement drainée par le cours d'eau en rabattant le niveau de la nappe sous celui du cours d'eau: phénomène dit de recharge induite. Le débit du cours d'eau s'en trouve d'autant diminué, ce qui contribue à aggraver les conséquences de la sécheresse observée à son niveau (cf. supra).

En outre il peut exister sur certains aquifères alluviaux et côtiers un impact qualitatif lié à la sécheresse.

Dans le cas d'alimentation, par le cours d'eau, de sa nappe d'accompagnement (recharge induite ou alimenta- tion naturelle) la dégradation de la qualité des eaux superficielles peut se répercuter sur celle des eaux souterraines par simple transfert.

Par ailleurs, le colmatage du lit du cours d'eau par de fines particules organiques, et le développement de conditions réductrices dans celui-ci peut induire une répercussion sur les eaux souterraines par augmentation de leur charge organique et installation de conditions anaérobies à l'interface nappe-cours d'eau: il peut en résulter dans la nappe et localement, la "libération " de formes réduites de métaux tels le fer et le manganèse, phénomène dont la relation avec les teneurs en matière organique a été démontrée.

Dans le cas de certains aquifères côtiers, les effets conjugués de la baisse de la ressource liée au déficit d'approvisionnement, et de la surexploitation peuvent favoriser l'intrusion du biseau salé, en bordure de côte, due au déplacement de l'équilibre des pressions entre les deux milieux, doux et salé.

Globalement toutefois, les eaux souterraines constituent des réserves naturelles moins vulnérables au déficit pluviométrique que les eaux superficielles et de plus grande capacité. L'exploitation rationnelle de leurs ressources permet de pallier aux carences périodiques des précipitations.

\section{Conclusion}

C'est principalement sur les milieux superficiels que l'impact de la sécheresse est le plus sensible. Quelle qu'en soit la saison il y a baisse immédiate de la capacité de dilution des rejets et de la valeur d'habitat vis-à-vis du poisson, par simple modification des caractéristiques physiques de l'écoulement. En ce qui concerne la dégradation de la capacité d'accueil des cours d'eau, à l'égard de la vie piscicole, il est important de noter que l'on dispose dorénavant d'outils qui permettent de prévoir, d'une manière spatialement fine les conséquences d'une baisse prolongée du débit.

A ceci s'ajoute, si la sécheresse est "chaude" un impact déterminant sur la prolifération végétale aggravée par le réchauffement des eaux.

Il en résulte toute une série de perturbations potentielles sur les processus physicochimiques et biochimiques qui confèrent au milieu une grande fragilité qui se manifeste par un comportement déséquilibré et incohérent, sur un cycle de temps très court, la journée, même si le bilan autoépuratoire peut, en certains cas, évoluer favorablement.

Les milieux saumâtres semblent affectés différemment selon qu'ils sont lagunaires ou estuariens. Les écosystèmes des milieux lagunaires sont, d'une certaine façon, accoutumés à de fortes variations de salinité par lesquelles se traduit principalement une réduction des apports d'eaux douces, de sorte que la capácitẻ de « récupération " de ces milieux est grande après la survenue d'un épisode prolongé de sécheresse.

En milieu estuarien, les conséquences jouent essentiellement au niveau de l'évolution du front de salinité et du bouchon vaseux: l'impact spatial est généralement étendu ce qui gêne considérablement les usages exigeants vis-à-vis de la salure de l'eau.

Ces milieux sont également très sensibles à l'eutrophisation et à cet égard, une perturbation de l'hydrologie en saison chaude induit comme dans le cas des milieux dulçaquicoles des proliférations végétales nuisibles à la pratique d'usages spécifiques à ces milieux comme la conchyliculture: plus qu'aux variations de salinité, à laquelle ils sont, dans une certaine mesure accoutumés, les bivalves sont sensibles aux conditions dégradées du milieu du point de vue de l'oxygène dissous, ou de l'élévation des concentrations de substances toxiques à leur égard, si leur taux dépasse certains seuils; ainsi qu'aux métabolites excrétés par certaines espèces de phytoplancton qui trouvent des conditions propices à leur développement explosif. Les produits de leur reproduction, particulièrement vulnérables aux conditions du milieu dans les premiers stades de leur vie ne peuvent accomplir leur développement normal.

Au total, ce sont les milieux souterrains et particulière- 
ment les aquifères de vaste extension spatiale, qui résistent le mieux à des sécheresses prolongées en raison de leur forte potentialité.

A ce niveau, les conséquences jouent principalement sur les échanges cours d'eau-nappes alluviales d'accompagnement, aux différents plans quantitatifs (d'autant qu'en période de sécheresse, la sollicitation est accrue) et, parfois, qualitatifs, la dégradation de la qualité du milieu courant se répercutant sur les eaux souterraines. Il est à noter que la saisonnalité de l'épisode joue un rôle important sur les conséquences différées dans le temps. A cet égard, les sécheresses «froides» présentent plus d'inconvénients que les sécheresses "chaudes" car elles jouent un rôle plus déterminant sur la recharge à terme des milieux et le bilan hydrique général.

En tout état de cause, la gestion de tels épisodes passe par :

1) une politique prévisionnelle cohérente d'aménagement des eaux permettant de suppléer au moins partiellement aux aléas climatiques. A cet égard, il est essentiel de préserver voire accroître la capacité naturelle du milieu à résister à un déficit prolongé de pluviométrie ;

2) une discipline dans la pratique des usages jouant essentiellement au niveau de l'utilisation de l'eau comme réceptacle des rejets. Les dispositifs épuratoires devraient être conçus de telle sorte qu'ils disposent de capacités accrues de stockage et gestion modulée des rejets mis en cuvre en période critique. De plus, différents niveaux de traitement devraient être prévus de manière à améliorer les rendements épuratoires lorsque les capacités réceptrices des milieux sont amoindries.

Enfin en matière d'alimentation en eau des populations, une diversification de l'approvisionnement est la meilleure parade à la raréfaction de la ressource. Les projets doivent être élaborés avec le souci d'assurer la sécurité quantitative (et qualitative aussi) ce qui passe par une prise en compte globale du problème et par l'élaboration de schémas d'alimentation en eau potable à une échelle territoriale idoine. Plus qu'en toute autre occasion, c'est au moment d'épisodes secs prolongés que la solidarité entre les usagers doit jouer.

\section{Bibliographie}

BÉDIOT, 1985: Faire face à la sécheresse. L'expérience française. La Houille blanche, $\mathrm{n}^{\circ}$ 6/7, 545-557.

Gosse et al., 1983: Les phénomènes d'aération dans le bilan d'oxygène dissous d'un cours d'eau aménagé. Rapport EDF HE/31-83.39-13 pages.

Gosse et SALleron, 1986 : Le rôle d'une chute d'eau dans le bilan d'oxygène dissous d'une rivière: application au barrage de Pont à Mousson sur la Moselle. XIX journées de l'Hydraulique de la SHF. Question IV, rapport n" 58 pages.

SRAE Franche Comté, 1988: Apparition de goûts et mortalités de poissons en relation avec le développement de populations d'algues dans les eaux du Doubs en amont de Montbeliard, 120 pages.

Travade, 1978: Enquête sur la sécheresse de 1976. Rapport EDF E31-78 N 19-16 pages + annexes.

CEMAGREF, 1989: Etude des conditions d'application de l'article 410 du Code Rural sur les débits réservés. Convention DPN n* 88-171-37 pages.

FRISONI, 1989: L'impact de la sécheresse sur les écosystèmes lagunaires du littoral méditerranéen. Réflexion théorique et présentation de quelques cas concrets. 5 pages.

Brossard et GallenNE, 1982: La qualité des eaux liées aux processus hydrosédimentaires dans l'estuaire de la Loire. TSM-l'eau, n* 12, 569-575.

Héral et al., 1978: Etude hydrobiologique du bassin de Marennes Oléron. Un exemple : la sécheresse de l'été 1976. Rev. Trav. Inst. Péchs. marit. 42(4) : 269-290.

Castany et Margat, 1976: La sécheresse de 1976 et les eaux souterraines. $P C M \mathrm{n}^{\circ}$ 5198-34-39, 